\title{
Radio observations of Galactic Center massive stars
}

\author{
Cornelia C. Lang \\ Astronomy Program, University of Massachusetts, \\ Amherst, MA 01003, USA
}

\begin{abstract}
High-resolution multi-frequency observations made with the VLA have revealed the presence of stellar winds in two of the three known massive stellar clusters at the Galactic Center (GC). At a distance of only $8.0 \mathrm{kpc}$, we are able to resolve radio sources associated with individual near-infrared sources known to be losing mass at high rates. Herein, I discuss the spectral and spatial properties of the radio sources and their near-infrared counterparts, their inferred mass-loss rates, variability and the prospects for detecting stellar winds sources in the Central Cluster, near the supermassive blackhole SgrA*. Such massive clusters as these three GC clusters have a profound impact on the interstellar medium, as evidenced by radio and X-ray observations of the surrounding regions.
\end{abstract}

\section{Introduction}

\subsection{The Galactic Center massive star population}

Over the last ten years, very high resolution near-infrared (near-IR) studies have revealed several extraordinary clusters of stars within the central $50 \mathrm{pc}$ of the Galaxy, where observations suffer 20-30 visual magnitudes of obscuration (e.g., Krabbe et al. 1995; Nagata et al. 1995; Cotera et al. 1996, 1999; Figer et al. 1999a,b): (i) the Central Cluster, located within a parsec of SgrA*; (ii) the Arches Cluster, located $\sim 30 \mathrm{pc} \mathrm{N}$ of SgrA* at $\ell=0.12, b=0.02$; and (iii) the Quintuplet Cluster, also located $\sim 35 \mathrm{pc} \mathrm{N}$ of SgrA*, at $\ell=0^{\circ} .16, b=0.06$. Many of the stars detected in these clusters have near-IR signatures of blue supergiants and late-type Wolf-Rayet stars, including WN, WC and WN9/Ofpe types, and most are thought to be losing mass from their surfaces at high rates. High-resolution radio continuum and recombination line studies of the unusuallyshaped filaments and streamers of ionized gas in the central $50 \mathrm{pc}$ of the Galaxy made with the Very Large Array of the National Radio Astronomy Observatory ${ }^{1}$ suggest that these regions are ionized by large numbers of young, massive stars (e.g., Lang, Goss \& Wood 1997; Lang, Goss \& Morris 2001a).

\subsection{Radio detections of stellar winds}

In addition to detecting the presence of massive stars based on the Lyman continuum flux they impart to adjacent $\mathrm{H}$ II regions, it is possible to detect the

\footnotetext{
${ }^{1}$ The National Radio Astronomy Observatory is a facility of the National Science Foundation operated under cooperative agreement by Associated Universities, Inc.
} 
expanding, ionized envelopes around individual hot stars. The classic theory of Panagia \& Felli (1975) and Wright \& Barlow (1975) predicts that the spectrum of radio emission from a stellar wind is $\propto \nu^{+0.6}$ for a spherically symmetric, isothermal, stationary wind expanding at a constant velocity. Previous surveys made with the VLA and the Australia Telescope Compact Array (ATCA) have detected centimeter radio continuum emission from OB supergiant and WolfRayet stellar winds in both the Northern and Southern skies (e.g., Abbott et al. 1986; Bieging et al. 1989; Leitherer, Chapman \& Koribalski 1995, 1997; Chapman et al. 1999).

The rising spectral index $(\alpha \simeq+0.6)$ is an important characteristic of radio detections of stellar winds, however, there is some variation in the observed spectra of stellar wind sources. About $25 \%$ of stellar wind detections have much flatter, or even nonthermal, spectra in the centimeter range (Bieging et al. 1989). The exact mechanism for producing such spectra is not well understood, but internal shocks in the wind of a single star (White 1985) has been suggested, and the presence of interacting winds in close binary systems (Williams et al. 1990, 1994; van der Hucht et al. 1992; Contreras \& Rodríguez 1999; Dougherty \& Williams 2000; Setia Gunawan et al. 2000, 2001) has been proven.

In addition to determining the spectral index of a stellar wind source, there are other important physical diagnostics that can be obtained through radio observations. The flux density of the stellar wind emission can be used to calculate the mass loss rate $(\dot{M})$. Based on Panagia \& Felli (1975), the mass loss rate can be estimated for observations at $8.5 \mathrm{GHz}$ using:

$$
\frac{\dot{M}}{10^{-5} \mathrm{M}_{\odot} \mathrm{yr}^{-1}}=0.52\left(\frac{S_{8.5}}{\mathrm{mJy}}\right)^{3 / 4}\left(\frac{v_{\infty}}{10^{3} \mathrm{~km} \mathrm{~s}^{-1}}\right)\left(\frac{d}{\mathrm{kpc}}\right)^{3 / 2}
$$

where $S_{8.5}$ is the flux density of the source at $8.5 \mathrm{GHz}, v_{\infty}$ is the terminal velocity of the wind, and $d$ is the distance to the source, i.e., $\sim 8 \mathrm{kpc}$ for the Galactic Center (GC). We have assumed an electron temperature of $10^{4} \mathrm{~K}, Z=1$, and a mean molecular weight, $\mu=2$, due to the enrichment in heavy elements of the late-type WN stars (Leitherer et al. 1997).

\section{Radio detections of Galactic Center stars}

The types of stars observed in the GC clusters should be losing mass at fairly high rates in expanding envelopes, and these stellar winds should be detectable at centimeter wavelengths. A number of detections of stellar wind sources in the GC clusters have been made to date using multi-frequency VLA observations. A summary of all observations is presented in Table 1. Initial results for the Quintuplet and Arches Clusters are published (Lang et al. 1999; Lang, Goss \& Rodríguez 2001b), but important follow-up work including pointed observations and searches for time-variability have been subsequently carried out. The focus of this paper is to discuss these newer results as well as the implications of such detections.

\subsection{The Arches Cluster}

Multi-frequency, multi-epoch radio observations of the Arches cluster (Figure 1) have revealed eight compact sources (AR1-AR5, AR7-AR9) with $\alpha \approx 0.3-0.9$ 
Table 1. Log of radio observations detected in Galactic Center clusters.

\begin{tabular}{lccrccl}
\hline \hline $\begin{array}{l}\text { source } \\
\text { name }\end{array}$ & $\begin{array}{c}\nu \\
(\mathrm{GHz})\end{array}$ & $\begin{array}{c}\text { VLA } \\
\text { array }\end{array}$ & $\begin{array}{r}\text { obs. } \\
\text { date }\end{array}$ & $\begin{array}{c}\text { resolution } \\
\left({ }^{\prime \prime}\right)\end{array}$ & $\begin{array}{c}\text { rms } \\
(\mathrm{mJy} \text { beam }\end{array}$
\end{tabular}

(Lang et al. 2001b). The nineth radio source, AR6, has a nonthermal spectrum $(\alpha=-0.7)$, which is consistent with the spectrum from $\sim 25 \%$ of radio stellar winds (Bieging et al. 1989). Seven of these nine sources have firm near-IR counterparts, of which the majority are Of/WN9 stellar types. The near-IR counterpart of AR6 lies outside the 1" positional error radius, and AR9 has no counterpart. These near-IR counterparts are known to be mass-losing emissionline stars (Nagata et al. 1995; Cotera et al. 1996). Therefore, the radio detections are thought to be detections of the stellar wind emission arising from these massive stars. The mass loss rates are calculated according to Equation 1, and they vary between $3-17 \times 10^{-5} \mathrm{M}_{\odot} \mathrm{yr}^{-1}$. Our second epoch $8.5 \mathrm{GHz}$ observations (January 2002) produced one new source, AR9, and three of the sources (AR1, AR4, AR7) showed variations in their flux densities of $10-35 \%$. This modest variability may be indicative of $(i)$ the presence of a time-variable non-thermal component (due to shocks in the wind; or rather colliding winds in binaries, see van der Hucht et al. 1992; Dougherty \& Williams 2000; Watson et al. 2002); and/or ( $i i)$ variability in the mass-loss rate or wind velocity.

\subsection{The Quintuplet Cluster}

Radio observations of the Quintuplet cluster have revealed eight compact sources (Figure 2). The initial observations of Lang et al. (1999) were pointed up to $4^{\prime}$ away from the cluster and did not have resolutions $<1^{\prime \prime}$, although six radio sources were detected. Three of these sources (QR4, QR5, and the Pistol Star) have near-IR massive star counterparts. Sources QR1-3 do not have counterparts and their nature is not as clear. It is possible that they represent enshrouded massive stars in the process of forming; their location at the edge of the cluster is also peculiar. The recent pointed observations (June 2002) had $0.5^{\prime \prime}$ resolution at $8.5 \mathrm{GHz}$ and revealed a total of eight radio sources, including five of the original sources. One of the original sources, QR4, did not appear, suggesting that it might be a time-variable source, as has now been detected in the Arches Cluster. Two additional sources, QR6 and QR7, appeared and have near-IR counterparts. The mass-loss rates implied for the Quintuplet Cluster sources are high - ranging from $1-20 \times 10^{-5} \mathrm{M}_{\odot} \mathrm{yr}^{-1}$. 


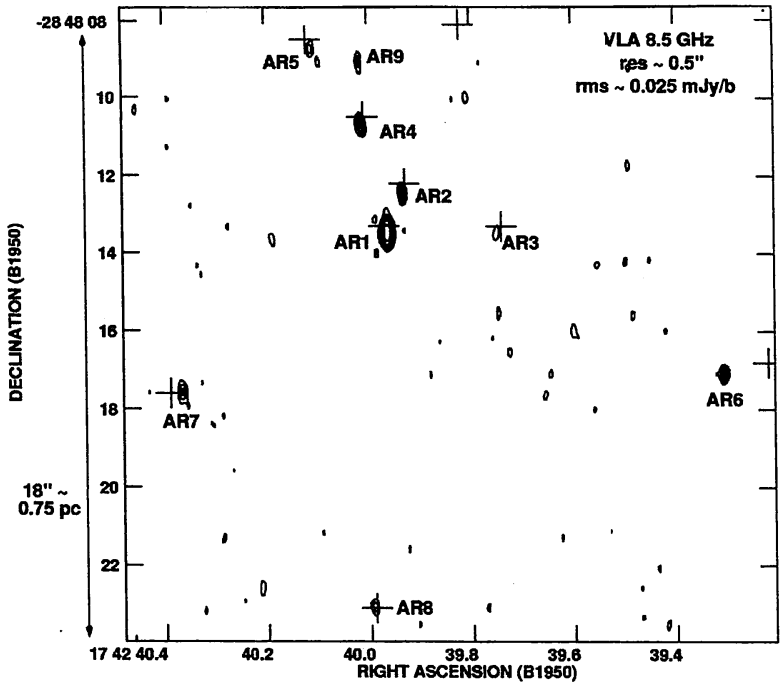

Figure 1. VLA $8.5 \mathrm{GHz}$ continuum image of the inner $16^{\prime \prime}(0.7 \mathrm{pc})$ of the Arches Cluster, with a resolution of $0^{\prime \prime} 41 \times 00^{\prime} 16, P A=2: 8$, and rms-noise of $25 \mu \mathrm{Jy}_{\text {beam }^{-1}}$. Contours correspond to $0.075,0.15,0.25,0.5,1.0,1.5,1.75$ $\mathrm{mJy}_{\text {beam }^{-1}}$. Crosses show positions of near-IR sources of Nagata et al. (1995), detected in $\mathrm{Br} \gamma$ and $\mathrm{Br} \alpha$ recombination lines.

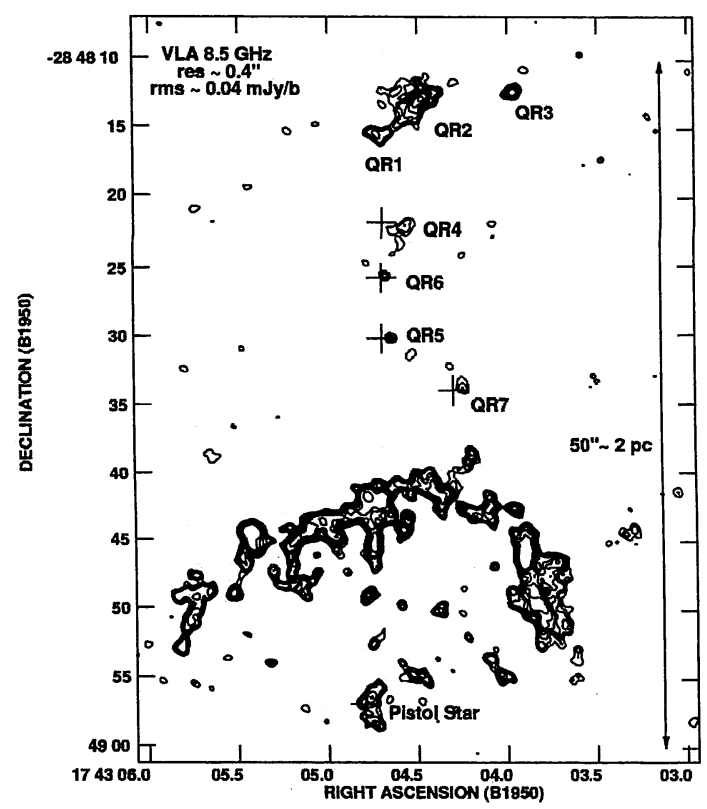

Figure 2. VLA $8.5 \mathrm{GHz}$ continuum image of the Pistol Star $\mathrm{H}$ II region and Quintuplet Cluster, with a resolution of $0.41 \times 0.16, P A=2.8$, and rms-noise of $0.1 \mathrm{mJy}_{\text {beam }}{ }^{-1}$. Contours correspond to $0.12,0.2,0.28,0.36,0.48,0.64$, $1.3,1.9 \mathrm{mJy}$ beam $^{-1}$. Crosses show positions of near-IR sources of Figer et al. (1999b). 
Table 2. Summary of radio sources detected in Galactic Center clusters.

\begin{tabular}{|c|c|c|c|c|c|c|c|}
\hline \multirow{2}{*}{$\begin{array}{l}\text { source } \\
\text { ID }\end{array}$} & \multicolumn{2}{|c|}{ other design. } & \multirow{2}{*}{$\begin{array}{c}S_{8.5} \mathrm{GHz} \\
(\mathrm{mJy})\end{array}$} & \multirow{2}{*}{$\begin{array}{c}S_{4.9} \mathrm{GHz} \\
(\mathrm{mJy})\end{array}$} & \multirow{2}{*}{$\begin{array}{l}\text { spectral } \\
\text { index } \alpha^{*}\end{array}$} & \multirow{2}{*}{$\begin{array}{c}\dot{M} \\
\left(\mathrm{M}_{\odot} \mathrm{yr}^{-1}\right)\end{array}$} & \multirow{2}{*}{$\begin{array}{l}\text { stellar type } \\
\text { counterpart }\end{array}$} \\
\hline & Nag95 & Cot96 & & & & & \\
\hline AR1 & \#8 & \#8 & $1.70 \pm 0.05^{\dagger}$ & $1.4 \pm 0.03$ & $0.35 \pm 0.04$ & $1.7 \times 10^{-4}$ & WN9/Ofpe ${ }^{a}$ \\
\hline AR2 & \#7 & $\# 6$ & $0.23 \pm 0.02$ & $<0.13$ & $>0.9$ & $3.9 \times 10^{-5}$ & WN8-9 \\
\hline AR3 & $\# 4$ & $\# 9$ & $0.17 \pm 0.02$ & $<0.13$ & $>0.4$ & $3.2 \times 10^{-5}$ & WN9/Ofpe \\
\hline AR4 & $\# 10$ & $\# 5$ & $0.17 \pm 0.02^{\S}$ & $0.16 \pm 0.03$ & $0.65 \pm 0.13$ & $3.9 \times 10^{-5}$ & WN9/Ofpe \\
\hline AR5 & $\# 11$ & $\# 2$ & $0.16 \pm 0.02$ & $<0.13$ & $>0.3$ & $3.0 \times 10^{-5}$ & WN8 \\
\hline AR6 & $\# 1$ ? & \#13? & $0.27 \pm 0.02$ & $0.40 \pm 0.03$ & $-0.7 \pm 0.08$ & $4.5 \times 10^{-5}$ & $\mathrm{WN} 8^{b}$ \\
\hline AR7 & \#14 & \#11 & $0.25 \pm 0.02^{\S}$ & $0.21 \pm 0.03$ & $0.31 \pm 0.05$ & $4.2 \times 10^{-5}$ & WN9/Ofpe \\
\hline AR8 & $\# 9$ & & $0.20 \pm 0.02$ & $<0.13$ & $>0.7$ & $3.6 \times 10^{-5}$ & no type \\
\hline \multirow[t]{2}{*}{ AR9 } & & & $0.17 \pm 0.04$ & $<0.13$ & $>0.4$ & $3.2 \times 10^{-5}$ & no cp. \\
\hline & Nag90 & Fig99 & & & & & \\
\hline $\mathrm{QR}^{c}$ & & & $2.0 \pm 0.3$ & $<1.5$ & $>0.5$ & $\ldots$ & no $\mathrm{cp}$. \\
\hline QR2 & & & $6.5 \pm 0.5$ & $3.0 \pm 0.5$ & $1.0 \pm 0.2$ & $\ldots$ & no cp. \\
\hline QR3 & & & $1.2 \pm 0.2$ & $1.2 \pm 0.2$ & $0.0 \pm 0.2$ & $\ldots$ & no $\mathrm{cp}$. \\
\hline QR4 & \#C & \#270N & $<0.15$ & $<0.30$ & $>-1$ & $1.4 \times 10^{-5}$ & $\mathrm{OBI}^{d}$ \\
\hline QR5 & $\# \mathrm{~F}$ & \#241 & $0.44 \pm 0.05$ & $<0.30$ & $>0.7$ & $6.5 \times 10^{-5}$ & WN9/Ofpe \\
\hline QR6 & & & $0.42 \pm 0.05$ & $<0.30$ & $>0.6$ & $6.1 \times 10^{-5}$ & no type \\
\hline QR7 & \#GCS3-2 & \#231 & $1.1 \pm 0.03$ & $<0.90$ & $>0.4$ & $1.3 \times 10^{-4}$ & DWCL? \\
\hline Pistol & & & $2.0 \pm 0.2$ & $4.7 \pm 0.5$ & $-1.7 \pm 0.3$ & $1.9 \times 10^{-4}$ & LBV \\
\hline
\end{tabular}

Notes: Cot96: Cotera et al. 1996; Fig99: Figer et al. 1999b; Nag90: Nagata et al. 1990; Nag95: Nagata et al. 1995.

* : where $S_{\nu} \propto \nu^{\alpha}$.

$\dagger: S_{8.5 \mathrm{GHz}}$ increased by $\sim 10 \%$ in second epoch (2002 Jan).

$\S: S_{8.5 \mathrm{GHz}}$ increased by $\sim 30 \%$ in second epoch (2002 Jan).

$a$ : spectral type from Cotera et al. (1996).

$b$ : identification with near-IR source not firm.

$c$ : all Quintuplet measurements from 2002 June 1 data.

$d$ : spectral type from Figer et al. (1999b).

\subsection{The Central Cluster}

Exploratory observations of the central parsec were made at $7 \mathrm{~mm}$ in the A-array in order to assess whether detections of any of the Central Cluster stellar winds would be possible. Detections of stellar wind emission at such close proximity to the central supermassive black hole, Sgr A*, would shed new light on the history of star-formation in this unique environment. The envelopes of highly evolved stars, such as IRS 7 and IRS 13, are known to be affected by their location in the central parsec. Our A-array observations showed Sgr A* as a strong source $(\sim 1 \mathrm{Jy})$, however, the sensitivity (even in a 4-hour observation) was not high enough for the dynamic range required to detect a $0.2 \mathrm{mJy}$ source.

\section{Implications of Galactic Center stellar wind detections}

The detection of stellar wind emission (and high mass loss rates) in the Arches and Quintuplet Clusters is one of the first detections of stellar wind emission from individual stars in a massive cluster. Further, the detection of stellar wind emission, coupled with the large ionizing fluxes of the clusters and the size of 


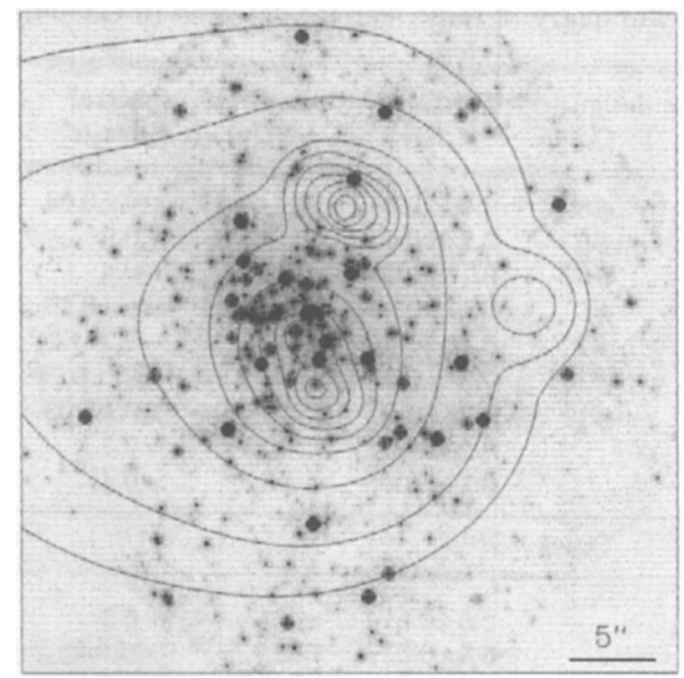

Figure 3. Overlay of contours of Chandra X-ray data on a HST-NICMOS image of the Arches Cluster (courtesy of A. Cotera).

the associated $\mathrm{H}$ II nebulae, makes them comparable to the starbursting regions NGC 3603 (Lang et al. 2001a; Moffat et al. 2002, and these Proceedings) and 30 Doradus in the LMC. Similarly, the rich population of massive stars in the GC region will have a profound impact on the interstellar environment. Ionization of the surfaces of dense molecular clouds and formation of the morphologicallyunique Arched Filaments and Sickle and Pistol H II nebulae have been shown to be due to the large ionizing fluxes of both the Arches and Quintuplet Clusters (Lang et al. 1997; Lang et al. 2001a; Figer et al. 1999a,b). Because of the denselypacked nature of these clusters, especially in the Arches Cluster (where the stellar density may exceed $\rho \approx 5 \times 10^{5} \mathrm{M}_{\odot} \mathrm{pc}^{-3}$ ), collisions between the expanding stellar winds may occur. Such collisions are thought to produce shocks which may create gas with temperatures as high as $10^{7} \mathrm{~K}$. Cantó et al. (2000) predict that such collisions may create a 'cluster wind', detectable in the X-ray regime. Raga et al. (2001) model the distribution of point-like and diffuse X-ray emission arising from clusters similar to the Arches Cluster.

The first high-resolution ( $\left.1^{\prime \prime}\right)$ X-ray observations of the Arches Cluster with Chandra reveal it to be one of the brightest X-ray sources in the GC region (Yusef-Zadeh et al. 2002; Wang, Gotthelf \& Lang 2002). Several point-like sources are detected in the cluster (see Figure 1) with $L_{\mathrm{x}} \approx 10^{35} \mathrm{erg} \mathrm{s}^{-1}$. These sources could represent colliding wind binaries of extremely massive stars in the Arches Cluster. The presence of strong diffuse X-ray emission from the central $50 \mathrm{pc}$ of the GC which has a spectrum consistent with $10^{7} \mathrm{~K}$ gas, suggests that much of the energetic activity in this region is driven by massive star formation activity (Wang, Gotthelf \& Lang 2002).

\section{References}

Abbott, D.C, Bieging, J.H., Churchwell, E., Torres, A.V. 1986, ApJ 303, 239 
Bieging, J.H., Abbott, D.C., Churchwell, E. 1989, ApJ 340, 518

Cantó, J, Raga, A., Rodríguez, L.F. 2000, ApJ 536, 896

Chapman, J.M., Leitherer, C., Koribalski, B., Bouter, R., Story, M. 1999, ApJ 518, 890

Contreras, M.E., Rodríguez, L.F. 1999, ApJ 515, 762

Cotera, A.S., Erickson, E.F., Colgan, S.W.J., et al. 1996, ApJ 461, 750

Cotera, A.S., Simpson, J.P., Erickson, E.F., et al. 1999, ApJ 510, 747

Dougherty, S.M., Williams, P.M. 2000, MNRAS 319, 1005

Figer, D.F., Kim, S.S., Morris, M., et al. 1999a, ApJ 525, 750

Figer, D.F., McLean, I.S., Morris, M. 1999b, ApJ 514, 202

van der Hucht, K.A., Williams, P.M., Spoelstra, T.A.Th., de Bruyn, A.G. 1992, in: L. Drissen, C. Leitherer \& A. Nota (eds.), Non-isotropic and Variable Outflows from Stars, ASP-CS 22, 253

Krabbe, A., Genzel, R., Eckart, A., et al. 1995, ApJ (Letters) 447, L95

Lang, C.C., Goss, W.M., Wood, D.O.S. 1997, ApJ 474, 275

Lang, C.C., Figer, D.F., Goss, W.M., Morris, M. 1999, AJ 118, 2327

Lang, C.C., Goss, W.M., Morris, M. 2001a, AJ 121, 2681

Lang, C.C., Goss, W.M., Rodgríguez, L.F. 2001b, ApJ (Letters) 551, L143

Leitherer, C., Chapman, J.M., Koribalski, B. 1995, ApJ 450, 289

Leitherer, C., Chapman, J.M., Koribalski, B. 1997, ApJ 481, 898

Moffat, A.F.J., Corcoran, M.F., Stevens, I.R., et al. 2002, ApJ 573, 191

Nagata, T., Woodward, C.E., Shure, M., Pipher, J.L., Okuda, H. 1990, ApJ 351, 83

Nagata, T., Woodward, C.E., Shure, M., Kobayashi, N. 1995, AJ 109, 1676

Panagia, N., Felli, M. 1975, A\&A 39, 1

Raga, A.C., Velázquez, P.F., Cantó, J., et al. 2001, ApJ 559, 33

Setia Gunawan, D.Y.A., de Bruyn, A.G., van der Hucht, K.A., Williams, P.M. 2000, A\&A 356, 676

Setia Gunawan, D.Y.A., de Bruyn, A.G., van der Hucht, K.A., Williams, P.M. 2001, A\&A 368, 484

Wang, Q.D., Gotthelf, E.V., Lang, C.C. 2002, Nature 415, 148

Watson, S.K., Davis, R.J., Williams, P.M., Bode, M.F. 2002, MNRAS 334, 631

White, R.L. 1985, ApJ 289, 698

Williams, P.M., van der Hucht, K.A., Pollock, A.M.T., et al. 1990, MNRAS 243, 662

Williams, P.M., van der Hucht, K.A., Spoelstra, T.A.Th. 1994, A\&A 291, 805

Wright, A.E., Barlow, M.J. 1975, MNRAS 170, 41

Yusef-Zadeh, F., Law, C., Wardle, M., et al. 2002, ApJ 570, 665

\section{Discussion}

KoenigsBerger: Weren't many of your mass-loss rates for the Arches Cluster stars $\left(\sim 10^{-5} \mathrm{yr}^{-1}\right)$ smaller than what Najarro presented?

LANG: I think that most of them are agreed. Yes, we are detecting mass loss rates for the same stars he is modeling.

CherepashchuK: Could you comment on the possible clumping structure of stellar winds, which should be taken into account for the correct determination of mass loss rates from observations of radio fluxes of hot stars? 
LANG: Currently, we have not taken any clumping factors into consideration for our mass loss calculations.

HILLIER: The influence of 'optically thick' clumps has to be considered. For continuum formation it is 'probably' not an issue. For line formation, the situation is more problematical. Of course, it depends on the clump properties, which are uncertain. If the clumps are thick and numerous, there is also an issue of driving them from the star.

LEITHERER: Can you say something about your non-detections? The upper limits are equally important for constraining mass loss rates, because they lower the mass loss rate averaged over the sample.

LANG: This is an important point which we need to explore further - you are right to point out that we are detecting the brightest (and therefore highest mass loss rate) sources in the cluster, and a more thorough analysis of our combined (two epoch) data on the Arches cluster will provide the full range of mass loss rates.

NAJARRO: Have you measured the mass loss rate for the dusty WC Wolf-Rayet stars? Is it consistent with other values from the literature?

LANG: The mass loss rates of the radio Quintuplet source QR 7, which appears to be related to the DWCL? type star (WCd?) is estimated to be $1.3 \times 10^{-4} \mathrm{M}_{\odot} \mathrm{yr}^{-1}$ from our $8 \mathrm{Ghz}$ observations.

DWARKADAS: You calculated the mass loss rates by assuming that all the emission was thermal. Yet you have mentioned that there is some variation in the spectra, which may be due to the presence of a non-thermal component. How have you taken the nonthermal flux into account, and how will that change the mass loss rates you quoted?

LANG: Only about $25 \%$ of our sources appear to have spectra flatter than $\nu^{+0.6}$, and we haven't currently taken the non-thermal component into consideration for calculation of the mass loss rate (where this might lead to overestimation of $\dot{M}$ ). Higher frequency $(7 \mathrm{~mm})$ observations are a good way to detect the thermal component. In one case (AR1) we do detect the wind emission at $7 \mathrm{~mm}$ and the mass loss rate based on the $7 \mathrm{~mm}$ flux density is $25 \%$ lower, so it needs to be considered.

KNÖDLSEDER: Concerning your X-ray data: you find that the previously claimed diffuse emission from the GC region is indeed partly due to point sources, which reduces the temperature from $10^{8} \mathrm{~K}$ to $10^{7} \mathrm{~K}$. Do you have information if this applies also to the Galactic ridge emission?

LANG: We would like to be able to address that important issue (the origin of the Galactic ridge emission), but we haven't gotten to it yet.

MARCHENKO: The young, compact cluster NGC 3603 is a good example of diffuse faint $\mathrm{X}$-ray emission. The emission is relatively soft, in the few-keV range. The phenomenon might be a common feature in young clusters.

LANG: Indeed, we do see a diffuse component of X-ray emission surrounding the Arches Cluster (in addition to several point-like sources). Unfortunately, the lack of sensitivity $<2 \mathrm{keV}$ prevents us from seeing an even softer diffuse component. 